\title{
Epidemiological and Clinical Aspects of Scorpion Sting among Children in South Part of Iran
}

\author{
Mehran Shahi ${ }^{1}$, Seyed H. Moosavy ${ }^{2}$, Javad Rafinejad ${ }^{3}$, Shahram Zare ${ }^{4}$, \\ Shahrokh Navidpour ${ }^{5} \&$ Abdoulhossain Madani ${ }^{6}$ \\ ${ }^{1}$ Infectious \& Tropical Diseases Research Center, Hormozgan University of Medical Sciences, Bandar Abbas, \\ Iran \\ ${ }^{2}$ Department of Internal Medicine, Infectious \& Tropical Diseases Research Center, Hormozgan University of \\ Medical Science, Bandar Abbas, Iran \\ ${ }^{3}$ Department of Medical Entomology and Vector Control, School of Public Health, Tehran University of Medical \\ Sciences, Tehran, Iran \\ ${ }^{4}$ Department of Community Medicine, Social Determinants on Health Promotion Research Center, Hormozgan \\ University of Medical Sciences, Bandar Abbas, Iran \\ ${ }^{5}$ Razi Reference Laboratory of Scorpion Research, Razi Vaccine and Serum Research Institute, Tehran, Iran \\ ${ }^{6}$ Department of Epidemiology, Social Determinants on Health Promotion Research Center, Hormozgan \\ University of Medical Sciences, Bandar Abbas, Iran \\ Correspondence: Abdoulhossain Madani, Department of Epidemiology, Social Determinants on Health Promotion \\ Research Center, Hormozgan University of Medical Sciences, Bandar Abbas, Iran. Tel: 98-917-166-4947. E-mail: \\ shmd_md@yahoo.com
}

Received: April 27, 2016 Accepted: June 27, 2016 Online Published: July 29, 2016

doi:10.5539/gjhs.v9n3p289 URL: http://dx.doi.org/10.5539/gjhs.v9n3p289

\begin{abstract}
Scorpion sting is regarded as one of the most medically-related problems in south part of Iran. This study aimed to describe the epidemiological aspects, clinical symptom and stinging agents among scorpion sting victims referred to Children Hospital in Hormozgan province. A cross-sectional study was conducted which involved all patients who had been stung by scorpion retaining scorpion sample at the time of admission. This study was conducted from May, 2014 to November, 2015. Data was gathered using the researcher made questionnaire and patient's fills. Data was analyzed by SPSS version 20, using descriptive and analytical statistics such as frequency, percentage, Chi-square and t-test. A total of 191 patients with age ranging from 1 month to 14 years (Mean=6 $6 \pm 2$ ) were studied. The most frequent patients were in the age group 6-10 years old. In this study 103 (53.9) were males and 88 (46.1) females. $169(88.5 \%)$ stung cases were from rural areas and $22(11.5)$ were reported from urban areas of the county. Most of the stings occurred indoors in rural areas. Most patients, $148(80.6 \%)$, had been admitted to emergency department within 1-6 hour after scorpion sting. The most prevalence of scorpion stings occurred on foot, 74 (38.7\%), and hand, 61 (31.9\%). Seventeen species belonging to two families Hemiscorpidae and Buthidae were identified. The main species of scorpion responsible for stinging was Hemiscorpius acanthocercus 41 (21.5\%) followed by Compsobuthus persicus, 37 (19.5), and H. enischnochela, 35 (18.4). The most common clinical symptoms observed in patients were pain (56.5\%), erythema (39.8\%) and burning sensation (33.5\%). The findings of this study, highlights the role of age, gender, residence location and scorpion species in envenomation. Therefore, health providers are recommended to consider these factors and the interference of these elements to prevent and care scorpion envenomation in children under 14 years old.
\end{abstract}

Keywords: epidemiology, symptom, scorpion, envenomation, Iran

\section{Introduction}

In the western and southern part of Iran, scorpion envenomation is one of the most critical medical problem leading to death, particularly, in children (Nejati, Mozafari, Saghafipour, \& Kiyani, 2014; Shahbazzadeh et al., 2009). In Iran, more than 75 percent of annual death due to scorpion sting occur in provinces including Khoozestan, SistanBaluchistan, Hormozgan and Kerman; most deaths occur in Khoozestan province (Kassiri et al., 2014). 
The risk of severe and fatal complications including developing severe cardiac, renal failure, respiratory and neurological complications in children associated with scorpion sting is actual in height (B. Vazirianzadeh, Farhadpour, Hosseinzadeh, Zarean, \& Moravvej, 2012). Factors that can increase the rate of scorpion sting severity are age, weight and duration of envenomation to the onset of treatment (Karnad, 2009).

The most noxious scorpions in the western and southwestern of Iran is Hemiscorpius. lepturus (Zare Mirakabadi, Mahmoodi Khatoonabadi, \& Teimoorzadeh, 2011). Hemiscorpius lepturus has cytotoxic venom that can cause severe complications such as, hemolysis, renal failure and probably leading to death (Dehghani, Khamechian, Vazirianzadeh, \& Moravvej, 2012; Jalali, Pipelzadedh, Sayedian, \& Rowan, 2010; Lowe, 2010; Pipelzadeh, Jalali, Pourabbas, \& Zaaremiraqabadi, 2007; Radmanesh, 1998).

According to the latest reports, Hormozgan Province has 20 scorpion species include: Hemiscorpius acanthocercus, H. enischnochela, H. lepturus, Razianus zarudnyi, Orthochirus farzanpayi, Or. varius, Or. stockwelli, Odontobuthus bidentatus, O. doriae, O. tavighiae, Mesobuthus phillipsii, M. eupeus persicus, Hottentotta saulcyi, Compsobuthus persicus, Compsobuthus plutenkoi, Sasanidotus gracilis, Iranobuthus krali, Buthacus macrocentrus, Androctonus crassicauda and Nebo henjamicus (Monod \& Lourenco, 2005; Navidpour, Soleglad, Fet, \& Kovarik, 2013).

The most dangerous scorpions in Hormozgan province are Hemiscorpius lepturus and H. acanthocercus that known by local names as Tekzard, Hash and Hoshkoo (Shahi, Azizi, \& Ansarian, 2009). A wide variety of clinical symptoms are manifested in patients who have had prior exposure to scorpion sting. These symptoms comprise, hyperthermia, irritability, profuse salivation, vomiting, tremor and convulsion leading to disorders of the nervous system, hemolysis, acute renal failure, cardiovascular disorders, edema, ecchymosis, blisters, local tissue destruction and even death (Rahmani et al., 2015). Few information is available concerning children envenomation due to scorpion stings in high risk areas such as Hormozgan province. Therefore, this study aimed at describing epidemiological characteristics and clinical symptom of scorpion envenomation among children in Hormozgan province, southern part of Iran.

\section{Materials and Methods}

This study was a cross-sectional research that analyzed the epidemiologic and clinical aspects of subjects who exposed to scorpion sting and referred to the Children Hospital of Bandar Abbas, which is reference as a teaching hospital for providing tertiary health care in south part of Iran, between May 2014 and November 2015.

We obtained demographic data, hospitalization term, sting time to hospital admission, sting site on the body, geographical locality of scorpion sting, scorpion sting place, anti-venoms used and scorpion species by filling a questionnaire after the purpose of the survey was explained. For clinical symptoms as well as history of treatment we used medical records in patient's fill. Data were analyzed by SPSS version 20 software and descriptive and analytical statistics such as frequency, percentage, Chi-square and t-test.

\subsection{Scorpion Identification}

Scorpion's samples were preserved in $75 \%$ ethanol and deposited in medical entomology laboratory of Bandar Abbas health school. Identification of scorpion species was carried out adopting morphological characteristics according to latest publication key diagnostics. Identification was accomplished using a Nikon XN model stereomicroscope. Publication of Monod \& Lourenco (2005), and Navidpour et al. (2013) was used as an identification key.

\section{Ethical Considerations}

This research was approved by ethical committee of Hormozgan University of Medical Sciences and all subjects filled the informed consent form.

\section{Results}

\subsection{Epidemiological Finding}

Totally, 191 subjects with scorpion on hand registered in the Bandar Abbas Children Hospital, over the one year of investigation, 2014-2015. The mean age of patients was 6 years old with standard deviation 2 years; and ranged from one month to 14 years. Majority of the cases were children between 6 and 10 years (37.0\%). There were 103 $(53.9 \%)$ male and $88(46.1 \%)$ female cases. Greatest cases occurred in rural, $169(88.5 \%)$, compared with urban, $22(11.5)$.

Indoor was prominent site, 112 (58.6\%) compared with outdoor site, 79 (41.4\%).

Results showed that more patients, $150(80.6 \%)$ had been admitted to emergency department within 1-6 hours after 
scorpion envenomation, followed by $19.4 \%$ who were referred within more than 6 hours (Figure 1).

Results of this study revealed that the most prevalence of scorpion stings occurred on foot 74 (38.7\%), followed by hand $61(31.9 \%)$, trunk $37(19.4 \%)$, head and neck $13(6.8 \%)$ and unknown $6(3.1 \%)$ respectively. All patients were treated with anti-venom serum. Total number of anti-venom vials used to treat all patients was 524 . The mean consumption per patient was 2.74 vials of anti-venom. The majority of patients $(108 ; 56.5 \%)$ were hospitalized for two days.

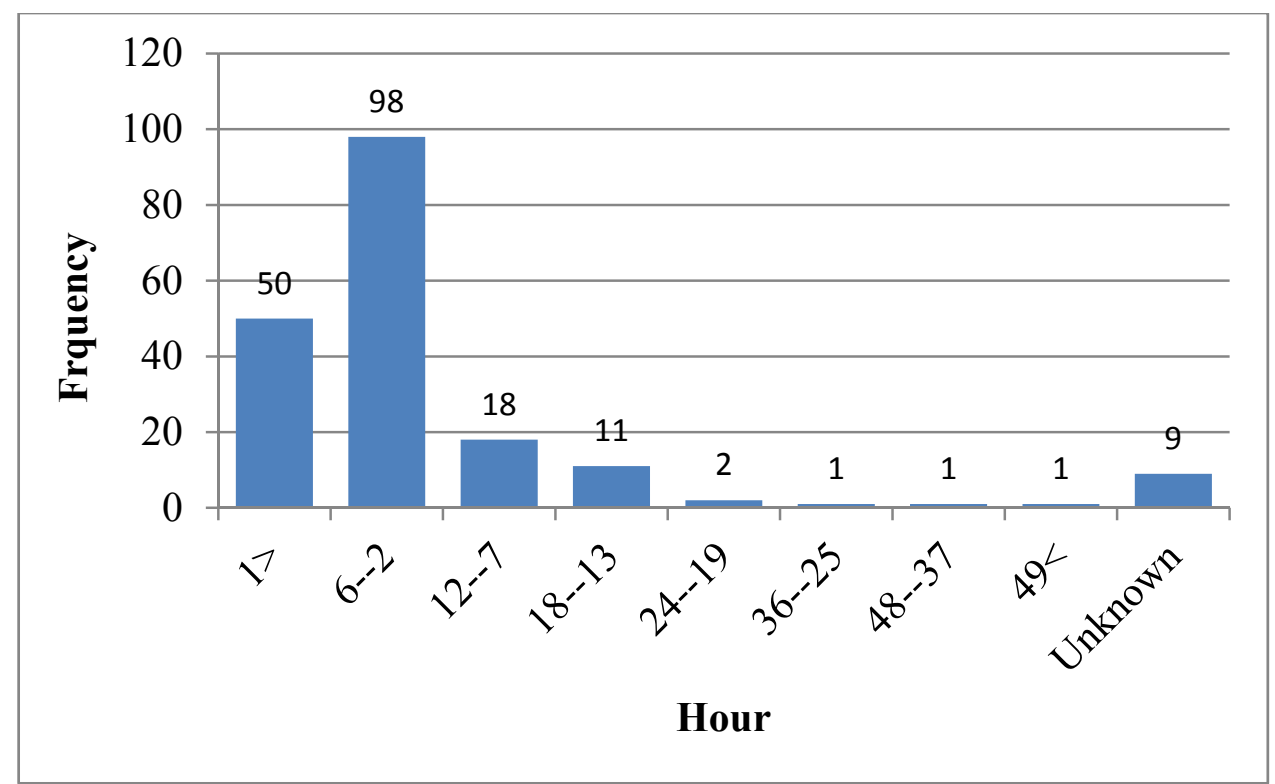

Figure 1. Frequency distribution of patients from time of sting to time of admission, south of Iran (2014-15)

\subsection{Scorpion Species}

Of 191 scorpion samples in which, were collected and laboratory analyzed, seventy species belonging to two families, Hemiscorpiidae and Buthidae, were identified; most of them depicting a yellow color.

The dominant species was Hemiscorpius acanthocercus (21.5\%) followed by Compsobuthus persicus, H. enischnochela, Mesobuthus persicus, Androctonus crassicauda, Odontobuthus doriae, Orthichirus stockwelli, M. phillipsii, Hottentotta Saulcyi, H. lepturus, H. gaillardia, H. persicus, O. brevedigitus, C. plutenkoi, C. matthiesseni, Or. varius and Razianus zarudnyi, respectively (Table 1).

Table 1. Frequency, percentage and color of scorpion species identified, south of Iran (2014-15)

\begin{tabular}{|c|c|c|c|c|}
\hline Family & Species & $\mathrm{N}$ & $\%$ & Scorpion color \\
\hline Hemiscorpiidae & H. acanthocercus & 41 & 21.5 & $\mathrm{Y}$ \\
\hline Buthidae & C. persicus & 37 & 19.5 & $\mathrm{Y}$ \\
\hline Hemiscorpiidae & H. enischnochela & 35 & 18.4 & $\mathrm{Y}$ \\
\hline Buthidae & M.eupeus persicus & 19 & 9.9 & $\mathrm{Y}$ \\
\hline Buthidae & A. crassicauda & 12 & 6.3 & $\mathrm{~B}$ \\
\hline Buthidae & O. doriae & 9 & 4.7 & $\mathrm{Y}$ \\
\hline Buthidae & Or. stockwelli & 9 & 4.7 & $\mathrm{~B}$ \\
\hline Buthidae & M. phillipsii & 7 & 3.7 & $\mathrm{Y}$ \\
\hline Buthidae & Ho. saulcyi & 6 & 3.1 & $\mathrm{Y}$ \\
\hline Hemiscorpiidae & H. lepturus & 5 & 2.6 & $\mathrm{Y}$ \\
\hline Hemiscorpiidae & H. gaillardi & 3 & 1.6 & $\mathrm{Y}$ \\
\hline
\end{tabular}




\begin{tabular}{lllll}
\hline Hemiscorpiidae & H. persicus & 2 & 1 & $\mathrm{Y}$ \\
Buthidae & O. brevidigitus & 2 & 1 & $\mathrm{Y}$ \\
Buthidae & C. plutenkoi & 1 & 0.5 & $\mathrm{Y}$ \\
Buthidae & C. matthiesseni & 1 & 0.5 & $\mathrm{Y}$ \\
Buthidae & R.zarudnyi & 1 & 0.5 & $\mathrm{Y}$ \\
Buthidae & Or. varius & 1 & 0.5 & $\mathrm{~B}$ \\
\hline & Total & 191 & 100 & \\
\hline
\end{tabular}

Note. $H=$ Hemiscorpius, $C=$ Compsobuthus, $M=$ Mesobuthus, Ho $=$ Hottentotta, $O=$ Odontobuthus, $R=$ Razianus, Or $=$ Orthochirus, $\mathrm{A}=$ Androctonus $\mathrm{N}=$ Number, $\%=$ Percentage, $\mathrm{Y}=$ yellow, $\mathrm{B}=$ black.

\subsection{Clinical Findings}

The most common clinical symptoms observed in patients included pain (56.5\%), erythema (39.8\%) and burning sensation $(33.5 \%)$. edema (16.8\%), warmth (13.1\%), nausea and vomiting $(10.5 \%)$, tenderness $(8.9 \%)$, itching $(8.4 \%)$, headache $(7.3 \%)$, dizziness $(6.3 \%)$, hematuria $(5.8 \%)$, urine color change $(5.8 \%)$, malaise $(5.8 \%)$, fever (5.2), necrosis (3.7\%) and abdominal pain (3.7\%) (Table 2).

Table 2. Frequency of clinical symptom following scorpion sting among 191 children less than 14 years, south of Iran (2014-15)

\begin{tabular}{lll}
\hline Symptom & $\mathrm{N}$ & $\%$ \\
\hline Pain & 108 & 56.5 \\
Erythema & 76 & 39.8 \\
Burning & 64 & 33.5 \\
Edema & 32 & 16.8 \\
Warmth & 25 & 13.1 \\
Nausea & 21 & 11 \\
Vomiting & 18 & 9.4 \\
Tenderness & 17 & 8.9 \\
Itching & 16 & 8.4 \\
Headache & 14 & 7.3 \\
Dizziness & 12 & 6.3 \\
Hematuria & 11 & 5.8 \\
Change the color of urine & 11 & 5.8 \\
Malaise & 11 & 5.8 \\
Fever & 10 & 5.2 \\
Necrosis & 7 & 3.7 \\
Abdominal pain & 7 & 3.7 \\
\hline
\end{tabular}

$\mathrm{N}=$ Number, $\%=$ percentage.

Other clinical signs observed with less frequency were sweat $(2.6 \%)$, cellulitis $(2.1 \% 0)$, tachycardia $(1 \%)$ and General rash $(0.5 \%)$. 


\section{Discussion}

Results of this study showed an increased risk of scorpion sting in the age group of 6 -10 years compared with other age groups. Similar results have been reported in the southeast Anatolia region of Turkey (Uluğ, Yaman et al. 2012). On the other hand, Adıgüzel et al. (Adiguzel, Ozkan et al. 2007) observed that children from 9 to 15 years old were more frequently affected than other age groups. Osnaya-Romero et al. (Osnaya-Romero, Mediana-Hernandez et al. 2001) stated that in Mexico, scorpion stings are mostly seen in patients aged 1-9. Different studies have shown varied age distributions for scorpion stings. Higher scorpion envenomation in this age group could be due to more physical activity and high-risk activities such as playing with stone and wood outdoors.

The finding in present study showed that male children were more involved than female. The results of Talebian (2006) study in Kashan denoted that scorpion sting in boys was more frequent (63\%) (Talebian \& Doroodgar, 2006). Similar findings are reported by Dehghani et al.(2010), Dabo et al.(2011), El Hidan et al. (2015), (Dabo et al., 2011; Dehghni, Vazirianzadeh, Rahimi-Nasrabadi, \& Moravvej, 2010; El Hidan, Oulaid Touloun, \& Boumezzough, 2015). This may be ascribed to vigorous activities engaged by boys, as compared to girls. Playing outside the homes increases the risk of scorpion stings in male children. However, the results of some studies are in contrast to our findings (Ozkan et al., 2006; Rahmani et al., 2015; B. Vazirianzadeh et al., 2012).

In this study, most scorpion sting cases occurred in rural areas compared with urban areas, which is in line with other studies conducted in Iran (Kassiri, Mohammadzadeh Mahijan, Hasanvand, Shemshad, \& Shemshad, 2012).However, high frequencies of the scorpion sting in the urban areas of the Khoozestan province had been reported as well (B. Vazirianzadeh et al., 2012). It can be inferred that this difference may be due to ecological factors, scorpion's fauna, distribution of scorpions and geographical differences in the western and southern regions of Iran.

Results of present research showed that most cases of scorpion sting occurred indoors. Similar to our results have been reported by Talebian and Dorodgar (2006), Behcet et al. (2009) and EL Hidan et al. (20015). In Sothern Iran, extreme heat provides conducive environment for scorpion invasion into susceptible houses. In effect, this is detrimental to possible contact with residing inhabitants.

We observed that only $21 \%$ of the patients were referred to health center during one hour after sting; while, further $80 \%$ of patients referred late (1 to 6 hours after scorpion envenomation). Apparently, lack of timely referral to medical center is reasonably due to lengthy routes and scarcity of access to vehicle. In furtherance, most scorpion stings usually occur during the second half of the night when people are asleep that results in the delay in referring patients to medical centers.

Significantly, the highest occurring site of scorpion sting was observed in the foot, hand and the trunk. This result is in line with other studies in Iran and Saudi Arabia (Kassiri, Mohammadzadeh Mahijan, et al., 2012), (Isazadehfar, Eslami, \& Entezariasl, 2013; Mahaba, 1997; B. Vazirianzadeh, Hossienzadeh, Moravej, Vazirianzadeh, \& Mosavi, 2013). The high ratio of scorpion stings in the foot and hand of children is due to walking barefooted, wearing inappropriate slipper and non-use of shoes at night, lifting stones by hand without any form of protection when playing. Moreover, most stings evident in the trunk of victims occurred during the time of sleep at night.

Our results showed that in this area the scorpion's fauna were two families, Hemiscorpiidae and Buthidae which consists of over 17 species. Of Hemiscorpiidae family, Hemiscorpius acanthocercus was the dominant species followed by H. enischnochela and of Buthidae family, C. persicus was the dominant. Scorpion fauna of Iran consists of 3 families Buthidae, Hemiscorpiidae and Scorpionidae which comprise 44 species in 23 genera (Kassiri et al., 2014) in which is almost in line of our results. In this contemporary study, H. gaillardi, H. persicus, C. matthiessen $i$ and $O$. brevedigitus are reported for the first time in Hormozgan province.

Comprehensively, 524 vials of scorpion anti-venom were used in the treatment of 191 patients. Considering accurate identification of scorpion species, it is probable that the use of this anti-venom is not practically necessary. Current polyvalent anti- venom contain six medically important scorpion species including, Androctonus crassicauda, Mesobuthus eupeus, H. saulcyi, Hottentotta schach, Odontobuthus doriae and H. lepturus (A. Jalali \& Rahim, 2014). According to a researcher, there is no need to use anti-venom for proper treatment of patients who have been stung by Buthidae scorpions. (Abourazzak et al., 2009; Abrough et al., 1999; Sofer, Shahak, \& Gueron, 1994). Unlike the above comments, some researchers recommend the use of anti-venom (Osnaya-Romero, Mediana-Hernandez, Florez-Hernandez, \& Leon-Rojas, 2001; Possani, 2000; Rahmani \& Jalali, 2012). 
Clinical condition of subjects in this study was not too much sever, however, most if victims developed clinical symptoms such as pain, erythema, burning and edema, which is in accordance with the findings of many of other studies (Kassiri, Teimouri, Shemshad, Sharifinia, \& Shemshad, 2012), (Rahmani et al., 2015), (Talebian \& Doroodgar, 2006).

Scorpion venom can cause a variety of sign such as pain at the site of sting, possible leading to death (Vatanpour, 2003). The results of Row et al.(2011) studies attested to the fact that Buthidae scorpions sting causes pain (Rowe et al., 2011). Buthidae scorpion's venom is a neurotoxin. This venom interrupts the nervous system causing pain and several other symptoms. Contrastingly, Hemiscorpiidae scorpion's sting results in less or no pain.

In our study the patients who were stung by Hemiscorius scorpions did not complain of pain at the beginning of sting, but after about 6 hours and more, swelling and pain at the sting site had developed. Hemiscorpius lepturus sting, does not cause immediate pain at the localized site of biting. It is likely that erythma and inflammation occur at the site of Hemiscorpius sting due to necrotizing property of its venom (Jalali et al., 2010; Pipelzadeh et al., 2007). However, delays in the treatment of these victims can cause hemolysis, hematuria, kidney failure, and even death. Other minor clinical symptoms were included sweat, cellulite, tachycardia and general rashes. No respiratory, cardiac and neurological complications in patients were observed.

\section{Conclusion}

The findings of this study, highlights the role of age, gender, residence location and scorpion`s species in envenomation as well as anti-venom therapy. Therefore, residents are recommended to consider these factors and the measures of environmental management for preventing scorpion bites. Moreover, health providers are recommended to update the clinical management to the patients due to misuse of anti-venom therapy.

\section{Acknowledgments}

This research work has been financed by Hormozgan University of Medical Sciences. We thank the participants who accepted to enter our study.

\section{Competing Interests Statement}

The authors declare that there is no conflict of interests regarding the publication of this paper.

\section{References}

Dabo, A., Golou, G., Traoré, M. S., Diarra, N., Goyffon, M., \& Doumbo, O. (2011). Scorpion envenoming in the north of Mali (West Africa): epidemiological, clinical and therapeutic aspects. Toxicon, 58(2), 154-158. http://dx.doi.org/10.1016/j.toxicon.2011.05.004

Dehghani, R., Khamechian, T., Vazirianzadeh, B. H., Vatandoost, \& Moravvej, S. A. (2012). Toxic Effects Of Scorpion, Hemiscorpius Lepturus (Hemiscorpiidae) Venom On Mice. 22(3), 593-596.

Dehghni, R., Vazirianzadeh, B., Rahimi-Nasrabadi, M., \& Moravvej, S. A. (2010). Study of scorpionism in Kashan in central of Iran. Pakistan J Med Sci, 26(10), 955-958.

El Hidan, M. A., Oulaid Touloun, O., \& Boumezzough, A. (2015). An epidemiological study of scorpion envenomation in the Zagora oases ( Morocco). Coastal Life Medicine, 3(9), 704-707.

Isazadehfar, K. H., Eslami, L., \& Entezariasl, M. (2013). Epidemiology of Scorpionism in southwest, Iran, 2008. Iranian Journal of Epidemiology, 8(4), 54-60.

Jalali, A., Pipelzadedh, M. H., Sayedian, R., \& Rowan, E. G. (2010). A review of epidemiological, clinical and in vitro physiological studies of envenomation by the scorpion Hemiscorpius lepturus (Hemiscorpiidae) in Iran. Toxicon 55, 173-179. http://dx.doi.org/10.1016/j.toxicon.2009.09.012

Karnad, D. R. (2009). Management of Scorpion Envenomation: Need For A Standard Treatment Protocol Using Drugs and Antivenom. JAPI, 57, 299-300.

Kassiri, H., Kassiri, A., Sharififard, M., Shojaee, S., Lotfi, M., \& Kasiri, E. (2014). Scorpion envenomation study in Behbahan County, Southwest Iran. Journal of Coastal Life Medicine, 2(5), 416-420.

Kassiri, H., Mohammadzadeh Mahijan, N., Hasanvand, Z., Shemshad, M., \& Shemshad, Kh. (2012). Epidemiological Survey on Scorpion Sting Envenomation in South-West, Iran. Zahedan J Res Med Sci., 14(8), 80-83.

Kassiri, H., Teimouri, A., Shemshad, M., Sharifinia, N., \& Shemshad, Kh. (2012). Epidemiological Survey and Clinical Presentation on Scorpionism in South-West of Iran. Middle-East Journal of Scientific Research, 12(3), 325-330. 
Lowe, G. (2010). Two new Hemiscorpius Peters, 1861 (Scorpiones: Hemiscorpiidae) from Northern Oman. Euscorpius, 91, 1-24.

Mahaba, H. M. A. (1997). Scorpion sting syndrome : epidemiology, clinical presentation and management of 2240 cases. Eastern Mediterranea Health Journal, 3(1), 82-89.

Monod, L., \& Lourenco, W. R. (2005). Hemiscorpiidae (Scorpiones) from Iran, with descriptions of two new species and notes on biogeography and phylogenetic relationships. Rev. Suisse Zool., 112(4), 869-941. http://dx.doi.org/10.5962/bhl.part.80331

Navidpour, Sh., Soleglad, M. E. , Fet, V., \& Kovarik, F. (2013). Scorpions of Iran (Arachnida, Scorpiones). Part IX. Hormozgan Province, with a Description of Odontobuthus tavighiae sp. n. (Buthidae). Euscorpius, 170, 1-29.

Nejati, J., Mozafari, E., Saghafipour, A., \& Kiyani, M. (2014). Scorpion fauna and epidemiological aspects of scorpionism in southeastern Iran. Asian Pac J Trop Biomed, 4(Suppl 1), S217-S221. http://dx.doi.org/10.12980/APJTB.4.2014C1323

Ozkan, O., Adiguzel, S., Yakistiran, S., Ylrm, C., Orman, M., \& Karaer, K. Z. (2006). Androctonus crassicauda (Olivier 1807) scorpionism in the Sanliurfa Provinces of Turkey. Acta Parasitol Turcica, 30, 239-245.

Pipelzadeh, M. H., Jalali, A., Pourabbas, M. T. R., \& Zaaremiraqabadi, A. (2007). An epidemiological and a clinical study on scorpionism by the Iranian scorpion Hemiscorpius lepturus. Toxicon, 50(7), 984-992. http://dx.doi.org/10.1016/j.toxicon.2007.07.018

Radmanesh, M. (1998). Cutaneous manifestation of the Hemiscorpius lepturus sting: A clinical study. Int $J$ Dermatol, 37(7), 500-507. http://dx.doi.org/10.1046/j.1365-4362.1998.00386.x

Rahmani, A. H., Forouzandeh, H., Kalantar, M., Asad-Masjedi, N., Alavian, Z., \& Kavarizadeh, K. (2015). Epidemiological and Clinical Characteristics of Scorpion Stings in Ahwaz, Southwest Iran (2006-2010). International Journal of Medical Toxicology and Forensic Medicine, 5(4), 201-206.

Rowe, A. H., Xiao, Y., Scales, J., Linse, K. D., Rowe, M. P., \& Zakon, H. H. (2011). Isolation and Characterization of CvIV4: A Pain Inducing a- Scorpion Toxin. PLoS ONE, 6(8), e23520. http://dx.doi.org/10.1371/journal.pone.0023520

Shahbazzadeh, D., Amirkhani, A., Djadid, N.D., Bigdeli, S., Akbari, A., Ahari, H., . . Dehgani, R. (2009). An epidemiological and clinical survey of scorpionism in Khuzestan province, Iran. Toxicon, 53(4), 454-459. http://dx.doi.org/10.1016/j.toxicon.2009.01.002

Shahi, M., Azizi, K., \& Ansarian, N. (2009). Study on Scorpion Fauna in endanger area of Hormozgan province 2006-2007. Journal of Hormozgan University of Medical Sciences and Health Services, 12(4), 207-214.

Talebian, A., \& Doroodgar, A. (2006). Epidemiologic study of scorpion sting in patients referring to Kashan medical centers during 1991-2002. Iranian Journal of Clinical Infectious Diseases, 1(4), 191-194.

Vatanpour, H. (2003). Effects of Black Scorpion Androctonus crasicuda Venom on Striated Muscle Preparation in vitro. Iranian Journal of Pharmaceutical Research, 17-22.

Vazirianzadeh, B., Hossienzadeh, M., Moravej, S. A., Vazirianzadeh, M., \& Mosavi, S. A. (2013). An Epidemiological Study on Scorpion Stings in Lordegan County, south-west of Iran. Archives of Razi Institute, 68(1), 71-76.

Vazirianzadeh, B., Farhadpour, F., Hosseinzadeh, M., Zarean, M., \& Moravvej, S. A. (2012). An Epidemiological and Clinical Study on Scorpionism in Hospitalized Children in Khuzestan, Iran. J Arthropod-Borne Dis, 6(1), 62-69.

Zare Mirakabadi, A., Mahmoodi Khatoonabadi, S., \& Teimoorzadeh, S. (2011). Antivenom injection time related effects of Hemiscorpius lepturus scorpion envenomation in rabbits. Archives of Razi Institute, 66(2), 139-145.

\section{Copyrights}

Copyright for this article is retained by the author(s), with first publication rights granted to the journal.

This is an open-access article distributed under the terms and conditions of the Creative Commons Attribution license (http://creativecommons.org/licenses/by/4.0/). 